to the operation, it was punctured to have the following medicine with a lancet, in order to see whether it was in a state of suppuration.

The cases mentioned last week, are going on very well.

A case of fractured clavicle, and leg, are the only accidents of importance that have been admitted this week.

ST. THOMAS'S IOSPITAL.

Amputation case continued.

Saturday, Nov. 29th. Passed a restless night; was delirious at times ; countenance sallow ; respiration 30 ; pulse 126 ; hiccough ; bowels open twice in the night; pain in epigastrio on pressure; tongue dry and brown; thirsty; has been very sick since yesterday, and vomited a good deal; striip pretty easy; outer part of the left thigh of a brownish hue; it became yesterday evening first $r 2 d$, then black, and afterwards of its present colour.

The following medicine was ordered for him yesterday afternoon :-

Be Decoct. Cinchonæ 3 jss.

Tinct. ejusdem $\breve{j}$ j.

- Opii m. v. M.

Fiat haustus ter die sumendus.

To day this was countermanded, and the patient was directed given him every four hours :-

B. Mist. Camp. 亏̆j.

Spirit, 瓦ther. Nitr. $3 j$. M.

A small quantity of castor, mixed up in a bolus, every four hours, was also prescribed. Pint of porter daily. Wine to be continued.

30th. Very restless in the mid. dle of the night ; has been dozing a good deal since; lies in that state with the eyes and mouth half open; countenance of a death-like appearance (facies hypocratica); pulse 120, rather full,--heart's action corresponding to the pulse; respiration sonorous, 42 ; hiccough still continues; bowels not open since the night before last; very thirsty; abdomen a little swollen, but not hard; not able to ask him any questions as he is asleep.

All the dressings were removed from the stump yesterday afternoon, the limb was well washed, two or three strips only of adhesive plaster were applied; to day the brownish appearance from the outside of the thigh is gone, but the stump is rather black, it feels easy; ordered to be kept constantly wet, with the spirit-wash, warmed.

R Continuentur medicamenta, cerevisia et vinum. 
At four in the afternoon his considerable quantity:of red frothy pulse began to fall, and at half mucus came out; the right past six p. m., he died.

\section{Inspectio cadaveris.}

The body was examined seventeen hours after death, by Mr. Babington, dresser to Mr. Travers, in the presence of two or three pupils.

The examination was made with such secrecy, that we were not able to be present; but from examining the body afterwards, we observed the following appearances :

External appearances.-Countenance sallow; back part of the right hand quite black; veins on the front of the abdomen very distinct, particularly on the left side, and of a blueish appearance; the cuticle removed for three or four inches in the neighbourhood of the stump, which was of a purplish hue.

Chest.-Effusion of a little more than half a pint of darkcoloured fluid into the right side of the chest; the lungs of this side were of a light grey marble colour anteriorly and externally, although when they were cut into, they were found to be red; posteriorly and laterally, of a livid appearance, and when divided a pleura costalis was red, but it did not adhere to the lungs - the lungs on the left side were more vascular anteriorly than those of the right: in the other parts they presented nearly the same appearances. There were two or three slight adhesions of the left pleura costalis to the pleura pulmonalis. Six ounces of dark-coloured fluid were effused into this side of the chest : in one word, the lungs were vascular throughout; but on the back part and sides they were gorged with blood, or bloody mucus. The bronchiæ contained some mucus: we believe that they were nearly full. The heart was very large, and more vascular than natural. The aorta and pulmonary arteries contained coagulated blood, or, at least, its fibrine part. The right auricle and venœ cavæ were filled with dark-coloured uncoagulated blood. The bronchial glands were considerably enlarged.

\section{Abdomen. - Liver was very} large and hard, of a yellowish appearance, both externally and internally : the gall-bladder contained a slight quantity of yellow bile. There were some livid spats on the outside of the stomach in 
several places: its internal mem- has had no unfavourable synpbrane was intensely inflamel. toms, and is at present rapidly The kidneys were more vascular recovering.

than natural, particularly the Noaccidents ofimportance have right. Bladder contained some been brought to this hospital arine, and was quite healthy.

The intestines presented no marks of inflammation.

The lower part of the aorta and vena cava, together with the iliac vessels, exhibited, on thtir inner surface, a different appearance from what is found in the healthy state; the arteries were slightly red, interrially, the veins livid; the nerves were healthy.

We were informed that there was a large quantity of matter formed near the stump; this, however, had been let out before we saw the body; there were evident marks of intense inflammation. The brain and spinal cord were not examined.

Before the accident, for nearly a twelve-month, he had been subject to a troublesome cough, and vomiting of blood.- 1 Coroner's inquest was held on the body, and the verdict returned was, Accidental Death.

Dec. 5. No operations have been performed here this week. stroyed by ulceration, which had The man from whom the stone extended down between the prewas removed last week, had a puce and body of the penis. Just slight bæmorrhage the same even- behind the glands, the urethra ing; but with this exception, he opened by a rery small orifice, 